\title{
Title: Canonical goal-selective representations are absent from prefrontal cortex in a spatial working memory task requiring behavioral flexibility
}

Authors: Claudia Böhm ${ }^{1 *}$, Albert K. Lee ${ }^{1 *}$

\author{
Affiliations: \\ ${ }^{1}$ Howard Hughes Medical Institute, Janelia Research Campus, Ashburn, VA 20147, USA \\ *Correspondence: boehmc@janelia.hhmi.org; leea@janelia.hhmi.org
}

\begin{abstract}
The prefrontal cortex (PFC)'s functions are thought to include working memory, as its activity can reflect information that must be temporarily maintained to realize the current goal. We designed a flexible spatial working memory task that required rats to navigate - after distractions and a delay - to multiple possible goal locations from different starting points and via multiple routes. This made the current goal location the key variable to remember, instead of a particular direction or route to the goal. However, across a broad population of PFC neurons, we found no evidence of current-goal-specific memory in any previously reported form - i.e. differences in the rate, sequence, phase or covariance of firing. This suggests such patterns do not hold working memory in the PFC when information must be employed flexibly. Instead, the PFC grouped locations representing behaviorally equivalent task features together, consistent with a role in encoding long-term knowledge of task structure.
\end{abstract}

Keywords: prefrontal cortex, working memory, flexible behavior, goal-directed behavior, spatial navigation, rat, mPFC, prelimbic, ACC, infralimbic, task structure, Neuropixels 


\section{Introduction}

Animals can pursue a goal while handling distractions and delays, starting from a variety of initial conditions, and adapting their responses in the face of unexpected obstacles. To guide such flexible goal-directed behavior, there needs to be a representation of the goal itself that is robust to these circumstances which can provide top-down instruction for selecting appropriate actions. The prefrontal cortex (PFC) plays a central role in flexible goal-directed behavior (Miller \& Cohen, 2001; Fuster, 2015), and one of its primary functions is thought to be the maintenance of information relevant for achieving the current goal (i.e. working memory) (Fuster \& Alexander, 1971; Funahashi et al., 1989; Miller et al., 1996; Rainer et al., 1998; Romo et al., 1999; Wang, 1999; Erlich et al., 2011; Wimmer et al., 2014; Inagaki et al., 2019; Wu et al., 2020). Therefore, the PFC is a prime candidate area for containing a representation of the current goal itself.

Spatial working memory tasks are well-suited for investigating such representations. First, the current goal, a particular spatial location, can be clearly specified and is ethologically relevant for many species (O'Keefe \& Nadel, 1978). Second, all of the aspects of flexibility mentioned above can be incorporated naturally in a form that many animals, including rodents, can solve. However, rodent spatial working memory experiments employed to date with recording in PFC or other brain areas (Wood et al., 2000; Frank et al., 2000; Baeg et al., 2003; Fujisawa et al., 2008; Gill et al., 2011; Harvey et al., 2012; Pfeiffer \& Foster, 2013; Wilkenheiser \& Redish, 2015; Ito et al., 2015; Spellman et al., 2015; Kim et al., 2016; Guise \& Shapiro, 2017; Bolkan et al., 2017) have not combined all of these elements of flexibility in a single task. As a result, potential working memory representations of the current goal have been difficult to dissociate from behavioral or sensory correlates. For instance, classic T-mazes have a single start and single route to each of the two goals. Thus, differential neural activity before reaching the $\mathrm{T}$ junction could represent the goals themselves, the two sets of stereotyped actions used to reach each goal as well as any associated sensory correlates (e.g. looking left versus right), or the plan to "go left" or "go right". Furthermore, any goal-specific activity could differ if the animal were to start from another point, and therefore not be usable under different initial conditions. Here, we devised a novel spatial working memory task incorporating multiple aspects of behavioral flexibility, allowing a search 
for a "pure" representation of the goal itself by disambiguating goal-related activity from other correlates.

\section{Results}

In our task, rats needed to remember one of three goal locations in each trial. The current goal was encoded during a "sample phase", in which animals were guided with light cues to one goal where they received a small reward. Rats had to remember this location until they needed to navigate to that goal again in the "test phase"-starting from one of three different locations and via one of three routes in the absence of lighted cues - to receive a large reward. The different routes were implemented by using an elevated maze design with bridges that could be raised (open) and lowered (blocked). The available route was only revealed after a $3 \mathrm{~s}$ (3.2 s in one animal) "fixation" period (hereafter referred to as the "delay period") during which animals had to hold their nose in one of the three test phase "start" location ports. The correct start location port was assigned pseudorandomly in each trial. To find the correct start port between each trial's sample and test phase, animals had to poke their nose into different ports until they found the one that elicited a tone when poked, which indicated the correct choice. (Figure 1A, B, Movie S1). The goal, sample phase route and test phase route were also pseudorandomly assigned in each trial (see methods). This design requires animals to update the currently relevant goal every trial (working memory task), and pushes them to remember the goal itself instead of memorizing a specific behavioral sequence or planning a particular motor action to reach the goal. Thus, the goal location is the key variable to retain, which must then be used flexibly to solve the task: navigating to that goal from any location by any route. Furthermore, having more than two goals excludes a strategy of navigating to one goal by default and only remembering when the goal is the other one-i.e. having three goals promotes the use of working memory representations of each goal itself.

Animals reached high levels of performance (Figure 1C, D, mean performance over all rats: $77.37,95 \% \mathrm{CI}$ : [72.46, 81.97]). To examine whether animals indeed remembered the goal location itself instead of particular routes, we compared the performance in trials where the route (bridge) animals had to take in the test phase was the same or different from the outbound and/or return bridge in the sample phase. The performance was comparable across routes for all animals, indicating that rats remembered goal locations instead of routes. Furthermore, the performance was comparable and above chance for all goal locations, implying that rats remembered each goal 
location rather than relying on a strategy of remembering only a subset of them. Rats spent several seconds in each task phase and generally ran faster on test outbound runs than sample outbound runs. The search time to find the correct start location was on average slightly higher in the trials where the subsequent choice was incorrect, but overlapped with search times in correct trials (Figure 1D).

A Neuropixels probe (Jun et al., 2017) was chronically implanted in medial prefrontal cortex (mPFC), previously shown to be required in variants of simpler rodent spatial working memory tasks (Spellman et al., 2015; Guise \& Shapiro, 2017). 100-200 units were simultaneously recorded across subareas including anterior cingulate (ACC), prelimbic (PL), infralimbic (IL), and dorsal peduncular (DP) cortex (Figure 1B) ( $\mathrm{n}=3$ animals; 182, 131, and 98 cells). We focused our analyses on the delay period since, during that time, behavior is well-controlled, and animals must remember a given goal while being in different, defined locations and facing different, defined directions, as well as not knowing the required future motor action. Analyses were applied to all putative principal cells with stable firing rates throughout performance $(97,84$, and 68 cells; see methods) and pooled across subareas (results including all cells or split by subarea were similar and are provided in the supplementary figures as indicated).

We searched for representations of the current goal encoded in terms of the major forms of delay period activity previously found in other working memory tasks, spatial or non-spatial, involving recordings from the PFC or elsewhere in primates and rodents: activity reflecting the representation at the time of encoding the sample item (Funahashi et al., 1989; Miller et al., 1996; Rainer et al., 1998; Romo et al., 1999; Wu et al., 2020), elevated/suppressed activity in single cells (Fuster \& Alexander, 1971; Funahashi et al., 1989; Miller et al., 1996; Rainer et al., 1998; Romo et al., 1999; Kim et al., 2016; Inagaki et al., 2019) or sequential firing patterns across multiple cells that tile the delay period (Baeg et al., 2003; Fujisawa et al., 2008; Pastalkova et al., 2008; Harvey et al., 2012; Ito et al., 2015), oscillatory phase-dependent firing (Siegel et al., 2009; Watrous et al., 2018), and elevated/suppressed covariances in firing among pairs of neurons (Barbosa et al., 2020).

We first tested whether memory of the current goal could be maintained during the delay period by a firing rate pattern across cells (population vector, PV) similar to the one when the animal was at the goal itself during the sample phase. To begin with, the PV at each goal during the sample phase was distinguishable and stable over time (Figures 2A and S2-1A), including 
across sample and test phases (Figure S2-1B). We correlated the overall activity during the delay period with the sample phase PV at each goal and asked whether it was more correlated with the currently remembered goal's PV. This was not the case (Figure 2B), as also seen in a different task (Guise \& Shapiro, 2017). To test if the remembered goal was represented as transient increases in correlation or a switching between current and other goals with the relevant (current) one being overrepresented (Kelemen and Fenton, 2010), we attempted to predict the goal based on correlation scores resulting from sliding a window of variable width across the delay period. While the animal's current (i.e. start) location was readily decodable (as expected), the remembered goal was not, using a wide range of time bins (Figure 2C) or activity restricted to individual subareas (Figure S2-2).

If the remembered goal is not maintained by activity directly related to activity at the goal itself, it could be (i) transformed into a different, but goal-specific, pattern, potentially dependent on the start location (ii) encoded in egocentric coordinates (i.e. the direction relative to the current start location) (Sarel et al., 2017) instead of in terms of the absolute (allocentric) goal location, (iii) represented by a sequential, instead of tonic, activity pattern and/or (iv) reflected in the phase of spike times or the short timescale interactions between pairs of neurons (Barbosa et al., 2020). We initially tested if any single cell activity in 100-ms- to full-delay-period-sized time bins showed consistent firing differences for allocentric, start-dependent or egocentric goal location (Figure S31). We found significant differences for the current (i.e. start) location, but not allocentric or egocentric goal location. There was also no evidence of start-dependent encoding of goals, i.e. a unique code for the 9 start-goal pairs. We then tested whether the remembered goal was encoded with a sequential activity pattern across multiple cells that may not be detectable at the single cell level (Figure 3). We employed several classification methods at multiple time resolutions (Figure 3C). Note that, for this analysis, potential activity patterns were always referenced to the delay period onset, as previously seen for memory-related sequences that tile the delay period (Fujisawa et al., 2008; Pastalkova et al., 2008; Harvey et al., 2012). Results were consistent across methods and time resolutions: current location could readily be decoded with any classification method for time bin resolutions between $100 \mathrm{~ms}$ to the full delay period duration. In contrast, no method could successfully classify the remembered goal in allocentric or egocentric coordinates at any time resolution (Figure 3C), including when using all cells regardless of firing rate stability (Figure S32). We next tested whether information might be stored in spike timing relative to local field 
potential (LFP) oscillations (Siegel et al., 2009; Watrous et al., 2018). We explored two frequency bands $(5-12 \mathrm{~Hz}, 15-30 \mathrm{~Hz})$, identified based on their power in the delay period. First, we calculated each cell's goal-specific phase preference in the delay period. We compared the distribution of phase preference magnitudes to one where goal labels were shuffled. The distributions were not different, suggesting the remembered goal does not affect overall phase preference. Second, we asked whether spike counts at specific phases might differ in a goal-specific manner, but they did not (Figures 4A and S4-1). We also explored the possibility of a recently described form of "activity-silent" memory, in which working memory is expressed in the spiking synchrony between pairs of neurons while stimulus information is not decodable from firing rates (Barbosa et al., 2020). However, neither for the pairs of neurons exhibiting excitatory interactions nor for the pairs exhibiting inhibitory interactions did covariances differ between trials associated with one goal versus the others (Fig 4B, and S4-2, see methods). Together, these results suggest that previously described forms of working memory maintenance are not responsible for storing the current goal in our task in which this information must be employed flexibly.

If mPFC does not encode memory of the goal in this task, what task-relevant processes might it support? We tested whether other task features could be decoded from mPFC activity. First, mPFC delay period activity did not indicate an upcoming or past error (Figure 5A), further corroborating that mPFC might not directly store current memory content. We then checked if mPFC distinguished the two task phases not only at the goal (Figure S2-1B) but when animals returned to the center. Before reaching the center, and at the center, task phase was not decodable (Figures 5B) (note the decodability afterwards could arise from cue or behavior differences in the two phases that were not present earlier). Lastly, we compared the population activity while rats engaged in different behaviors at different locations. Within each group of behaviors (i.e. waiting at a start nose port, crossing a bridge/route, consuming reward at a goal), mPFC displayed spatial selectivity (e.g. it differentiated the three bridges). Furthermore, we found that this spatial selectivity was embedded within a larger organization of activity in which these distinct, taskrelevant groups were clearly separable from each other (Figures 5C and S5). 


\section{Discussion}

Previous work has shown sensory stimulus-specific delay period activity (“delay activity”) independent of motor plans (Romo et al., 1999; Wu et al., 2020) or resistant to distractor stimuli (Miller et al., 1996), and start-independent spatial (Brown et al., 2016; Guise \& Shapiro, 2017; Watrous et al., 2018) or route-independent visuospatial (Saito et al., 2005) goal-specific delay activity in the PFC. A pair of studies (using a single start location) (Spellman et al., 2015; Bolkan et al., 2017) showed no evidence of goal-selective delay activity in rodent prelimbic mPFC. In one of these studies, motor planning was prevented, but not in the other (i.e. standard T-maze), and a study similar to the latter one (Kim et al., 2016) found goal-selective delay activity. Another study (Lara \& Wallis, 2014) found no evidence of visual stimulus-selective delay activity in primate PFC. However, this study used color as the relevant stimulus dimension and found little evidence of color-selective activity even during stimulus presentation. Since PFC neurons have been shown in other cases to encode sample stimulus color (Buschman et al., 2012), this suggests that encoding of the stimulus during the sample period may be a prerequisite of observing stimulus-selective activity in the delay period. Spatial information is strongly represented in PFC in primates (Funahashi et al., 1989; Rainer et al., 1998; Saito et al., 2005; Lara \& Wallis, 2014) and essentially all rodent studies, including here (Figures 2A and S2-1); yet, we found no spatial goal-selective delay activity. Furthermore, our ability to decode the current start position, and the relatively high number of cells recorded simultaneously in our study, suggest that our data set was large enough to have detected an effect of sizes previously reported in the literature for simpler tasks.

In contrast to previous studies, we combined all elements of flexibility in one task distractions, different start locations, and different, unpredictable routes, as well as more than two goals. We found no evidence of goal-selective delay activity in any of the major forms previously documented over a wide range of parameters and across large numbers of simultaneously recorded cells in multiple mPFC subareas. Thus, these representations are unlikely to serve as general working memory correlates that can be employed under conditions of high behavioral flexibility, such as those often encountered in the real world. Whether animals in simpler tasks use such canonical representations of working memory to solve those tasks, employ an alternative strategy, or rely on an as-yet-undiscovered pattern of activity remains an open question. Our work therefore stresses the importance of employing more cognitively challenging tasks that allow dissociation 
between correlates of high-level cognitive variables and other task-related variables. The search for a pure, flexible working memory correlate could focus both on other brain areas, or on exploring as-yet-unobserved activity patterns or alternative memory mechanisms involving the mPFC - for instance, those related to short-term synaptic plasticity mechanisms (Mongillo et al., 2008) beyond the previously reported covariance patterns (Barbosa et al., 2020) investigated here. Finally, our results suggest a role for mPFC in working memory tasks by representing task structure in terms of groups of behaviorally related elements (Jung et al., 1998; Yu et al., 2018; Kaefer et al., 2020), consistent with findings that the PFC forms long-term memories of learned stimulus categories (Freedman et al., 2001).

\section{Acknowledgments}

This work was funded by the Howard Hughes Medical Institute. We thank S. Erwin, R. Gattoni, P. Rich, J. Jun, B. Karsh, J. Colonell, B. Barbarits, W. Sun, T. Harris, J. Chen, J. Arnold, S. Sawtelle, P. Polidoro, Vidrio, S. Romani, and K. Branson for assistance and advice. We thank A. Hermundstad, A. Hantman, S. Romani, W. Asaad, A. Dorrn, and J. Dudman for comments on the manuscript.

\section{Author contributions}

C.B. and A.K.L. designed the study. C.B. performed experiments and analyzed data, with input from A.K.L. C.B. and A.K.L. wrote the paper. 


\section{References}

Baeg, E. H., Kim, Y. B., Huh, K., Mook-Jung, I., Kim, H. T. \& Jung, M. W. (2003). Dynamics of population code for working memory in the prefrontal cortex. Neuron, 40, 177-188.

Barbosa, J., Stein, H., Martinez, R.L., Galan-Gadea, A., Li, S., Dalmau, J., Adam, K. C., Valls-Solé, J., Constantinidis, C. \& Compte, A. (2020). Interplay between persistent activity and activity-silent dynamics in the prefrontal cortex underlies serial biases in working memory. Nat Neurosci, 23, 1016-1024.

Bolkan, S. S., Stujenske, J. M., Parnaudeau, S., Spellman, T. J., Rauffenbart, C., Abbas, A. I., Harris, A. Z., Gordon, J. A. \& Kellendonk, C. (2017). Thalamic projections sustain prefrontal activity during working memory maintenance. Nat Neurosci, 20, 987-996.

Brown, T. I. et al. (2016). Prospective representation of navigational goals in the human hippocampus. Science, 352, 1323-1326.

Buschman, T. J., Denovellis, E. L., Diogo, C., Bullock, D. \& Miller, E. K. (2012). Synchronous oscillatory neural ensembles for rules in the prefrontal cortex. Neuron, 76, 838-846.

Erlich, J. C., Bialek, M. \& Brody, C. D. (2011). A cortical substrate for memory-guided orienting in the rat. Neuron, 72, 330-343.

Frank, L. M., Brown, E. N. \& Wilson, M. (2000). Trajectory encoding in the hippocampus and entorhinal cortex. Neuron, 27, 169-178.

Freedman, D. J., Riesenhuber, M., Poggio, T. \& Miller, E. K. (2001). Categorical representation of visual stimuli in the primate prefrontal cortex. Science, 291, 312-316.

Fujisawa, S., Amarasingham, A., Harrison, M. T. \& Buzsáki, G. (2008). Behavior-dependent short-term assembly dynamics in the medial prefrontal cortex. Nat Neurosci, 11, 823-833.

Funahashi, S., Bruce, C. J. \& Goldman-Rakic, P. S. (1989). Mnemonic coding of visual space in the monkey's dorsolateral prefrontal cortex. J Neurophysiol, 61, 331-349.

Fuster, J. M. (2015). The Prefrontal Cortex. 5th ed. Academic Press.

Fuster, J. M. \& Alexander, G. E. (1971). Neuron activity related to short-term memory. Science, 173, 652-654.

Gill, P. R., Mizumori, S. J. Y. \& Smith, D. M. (2011). Hippocampal episode fields develop with learning. Hippocampus, 21, 1240-1249.

Guise, K. G. \& Shapiro, M. L. (2017). Medial Prefrontal Cortex Reduces Memory Interference by Modifying Hippocampal Encoding. Neuron, 94, 183-192.

Harvey, C. D., Coen, P. \& Tank, D. W. (2012). Choice-specific sequences in parietal cortex during a virtualnavigation decision task. Nature, 484, 62-68.

Inagaki, H. K., Fontolan, L., Romani, S. \& Svoboda, K. (2019). Discrete attractor dynamics underlies persistent activity in the frontal cortex. Nature, 566, 212-217. 
Ito, H. T. et al. (2015). A prefrontal-thalamo-hippocampal circuit for goal-directed spatial navigation. Nature, 522, 50-55.

Jung, M. W., Qin, Y., McNaughton, B. L. \& Barnes, C. A. (1998). Firing characteristics of deep layer neurons in prefrontal cortex in rats performing spatial working memory tasks. Cereb Cortex, 8, 437-450.

Jun, J. J. et al. (2017). Fully integrated silicon probes for high-density recording of neural activity. Nature, $551,232-236$.

Jun, J. J., Mitelut, C., Lai, C., Gratiy, S. L., Anastassiou, C. A., Harris, T. D. (2017). Real-time spike sorting platform for high-density extracellular probes with ground-truth validation and drift correction. https://www.biorxiv.org/content/10.1101/101030v2.

Kaefer, K., Nardin, M., Blahna, K. \& Csicsvari, J. (2020). Replay of behavioral sequences in the medial prefrontal cortex during rule switching. Neuron, 106, 154-165

Kelemen, E. \& Fenton, A. A. (2010). Dynamic grouping of hippocampal neural activity during cognitive control of two spatial frames. PLoS Biol, 8, e1000403.

Kim, D. et al. (2016). Distinct Roles of Parvalbumin- and Somatostatin-Expressing Interneurons in Working Memory. Neuron, 92, 902-915.

Lara, A. H. \& Wallis, J. D. (2014). Executive control processes underlying multi-item working memory. Nat Neurosci, 17, 876-883.

Maaten, L. v. d. \& Hinton, G. (2008). Visualizing data using t-SNE. Journal of machine learning research, 9, 2579-2605.

Miller, E. K. \& Cohen, J. D. (2001). An integrative theory of prefrontal cortex function. Annu Rev Neurosci, 24, 167-202.

Miller, E. K., Erickson, C. A. \& Desimone, R. (1996). Neural mechanisms of visual working memory in prefrontal cortex of the macaque. J Neurosci, 16, 5154-5167.

Mongillo, G., Barak, O. \& Tsodyks, M. (2008). Synaptic theory of working memory. Science, 319, 15431546.

O'Keefe, J. \& Nadel, L. (1978). The Hippocampus as a Cognitive Map. Oxford University Press.

Pastalkova, E., Itskov, V., Amarasingham, A. \& Buzsáki, G. (2008). Internally generated cell assembly sequences in the rat hippocampus. Science, 321, 1322-1327.

Pedregosa, F. et al. (2011). Scikit-learn: Machine learning in Python. Journal of Machine Learning Research, 12, 2825-2830.

Pfeiffer, B. E. \& Foster, D. J. (2013). Hippocampal place-cell sequences depict future paths to remembered goals. Nature, $497,74-79$.

Rainer, G., Asaad, W. F. \& Miller, E. K. (1998). Selective representation of relevant information by neurons in the primate prefrontal cortex. Nature, 393, 577-579. 
Romo, R., Brody, C. D., Hernández, A. \& Lemus, L. (1999). Neuronal correlates of parametric working memory in the prefrontal cortex. Nature, 399, 470-473.

Saito, N. et al. (2005). Representation of immediate and final behavioral goals in the monkey prefrontal cortex during an instructed delay period. Cereb Cortex, 15, 1535-1546.

Sarel, A., Finkelstein, A., Las, L. \& Ulanovsky, N. (2017). Vectorial representation of spatial goals in the hippocampus of bats. Science, 355, 176-180.

Siegel, M., Warden, M. R. \& Miller, E. K. (2009). Phase-dependent neuronal coding of objects in shortterm memory. Proc Natl Acad Sci USA, 106, 21341-21346.

Spellman, T. et al. (2015). Hippocampal-prefrontal input supports spatial encoding in working memory. Nature, 522, 309-314.

Wang, X. J. (1999). Synaptic basis of cortical persistent activity: the importance of NMDA receptors to working memory. J Neurosci, 19, 9587-9603.

Watrous, A. J. et al. (2018). Phase-tuned neuronal firing encodes human contextual representations for navigational goals. eLife, 7 , e32554.

Wikenheiser, A. M. \& Redish, A. D. (2015). Hippocampal theta sequences reflect current goals. Nat Neurosci, 18, 289-294.

Wimmer, K., Nykamp, D. Q., Constantinidis, C. \& Compte, A. (2014). Bump attractor dynamics in prefrontal cortex explains behavioral precision in spatial working memory. Nat Neurosci, 17, 431-439.

Wood, E. R., Dudchenko, P. A., Robitsek, R. J. \& Eichenbaum, H. (2000). Hippocampal neurons encode information about different types of memory episodes occurring in the same location. Neuron, 27, 623633.

Wu, Z., Litwin-Kumar, A., Shamash, P. Taylor, A., Axel, R. \& Shadlen, M. N. (2020). Context-dependent decision making in a premotor circuit. Neuron, 106, 316-328.

Yu, J. Y. et al. (2018). Specific hippocampal representations are linked to generalized cortical representations in memory. Nat Commun, 9, 2209. 


\section{Figure 1}

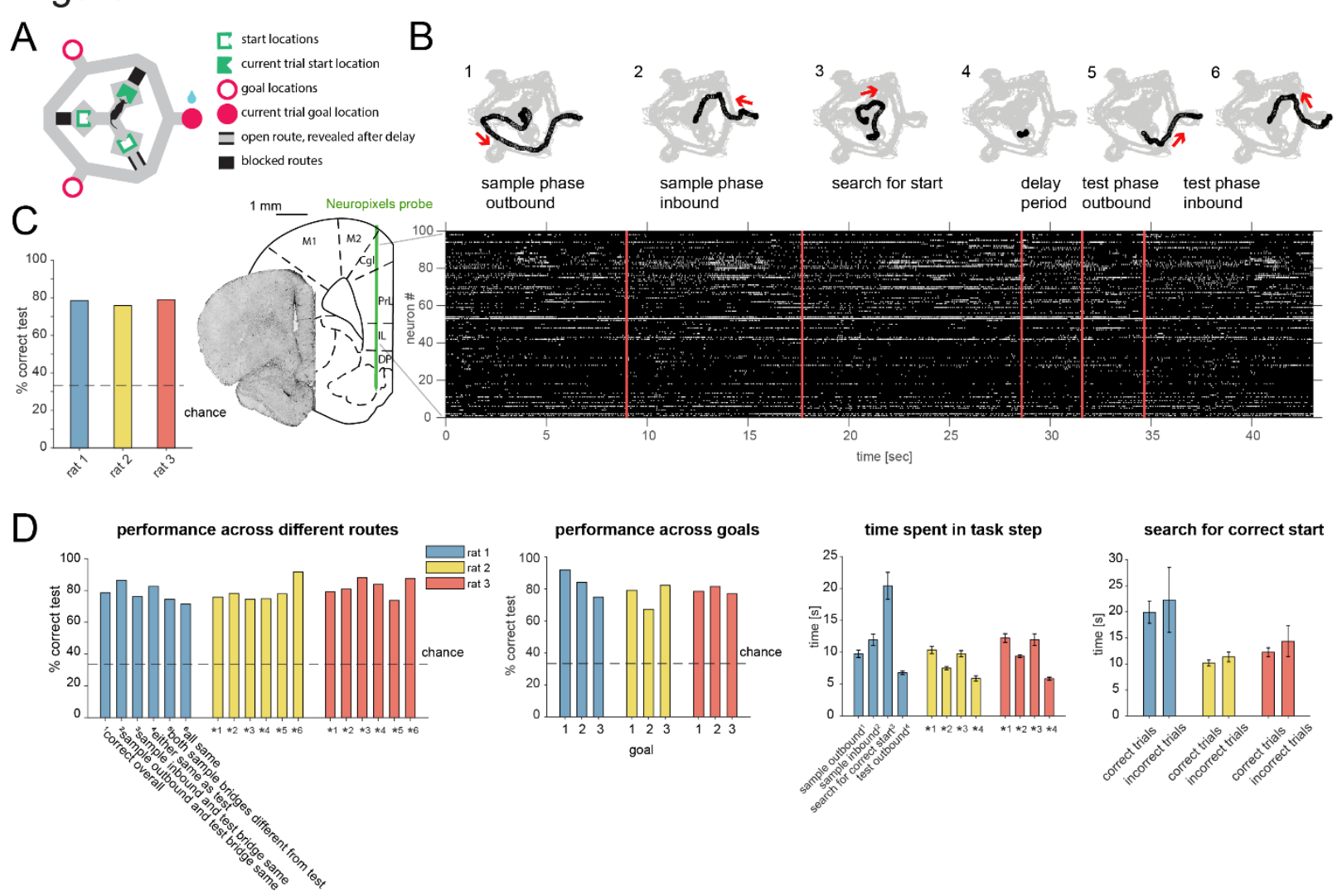

Figure 1. Task design, behavior and recording.

(A) Schematic of "multi-start/multi-goal/multi-route" ("MSMGMR") task environment. (B) Top: Each trial consisted of the following steps: (1) the current goal is randomly assigned and cued with lights; a single, randomly assigned route (bridge) is available; rat gets small reward upon arrival at cued goal; (2) animal returns to center via any route; all routes are blocked upon arrival at center; (3) animal searches for randomly assigned start position, indicated by a tone once animal pokes nose into correct port; (4) animal must maintain nose poke for $3 \mathrm{~s}$; (5) a randomly assigned route becomes available and animal can navigate to goal; (6) animal returns to center via any route to initiate next trial (see Movie S1). Bottom left: Neuropixels probe is chronically implanted in mPFC, and 384 channels spanning multiple subareas are recorded from simultaneously. Bottom right: Spiking activity during task. (C) Task performance (test phase). (D) Left: Task performance in subsets of trials in which different routes were taken. Again, a single outbound route was randomly assigned for both test and sample phases; inbound routes could be freely chosen by the animal. Center left: Performance for each goal location. Center right: Time spent for each of the 
bioRxiv preprint doi: https://doi.org/10.1101/2020.09.11.292888; this version posted September 11, 2020. The copyright holder for this preprint (which was not certified by peer review) is the author/funder, who has granted bioRxiv a license to display the preprint in perpetuity. It is made available under aCC-BY-NC-ND 4.0 International license.

task steps (see also B) (mean and 95\% CI). Right: Search time for the start location for correct and incorrect trials (mean and $95 \% \mathrm{CI}$ ). 


\section{Figure 2}

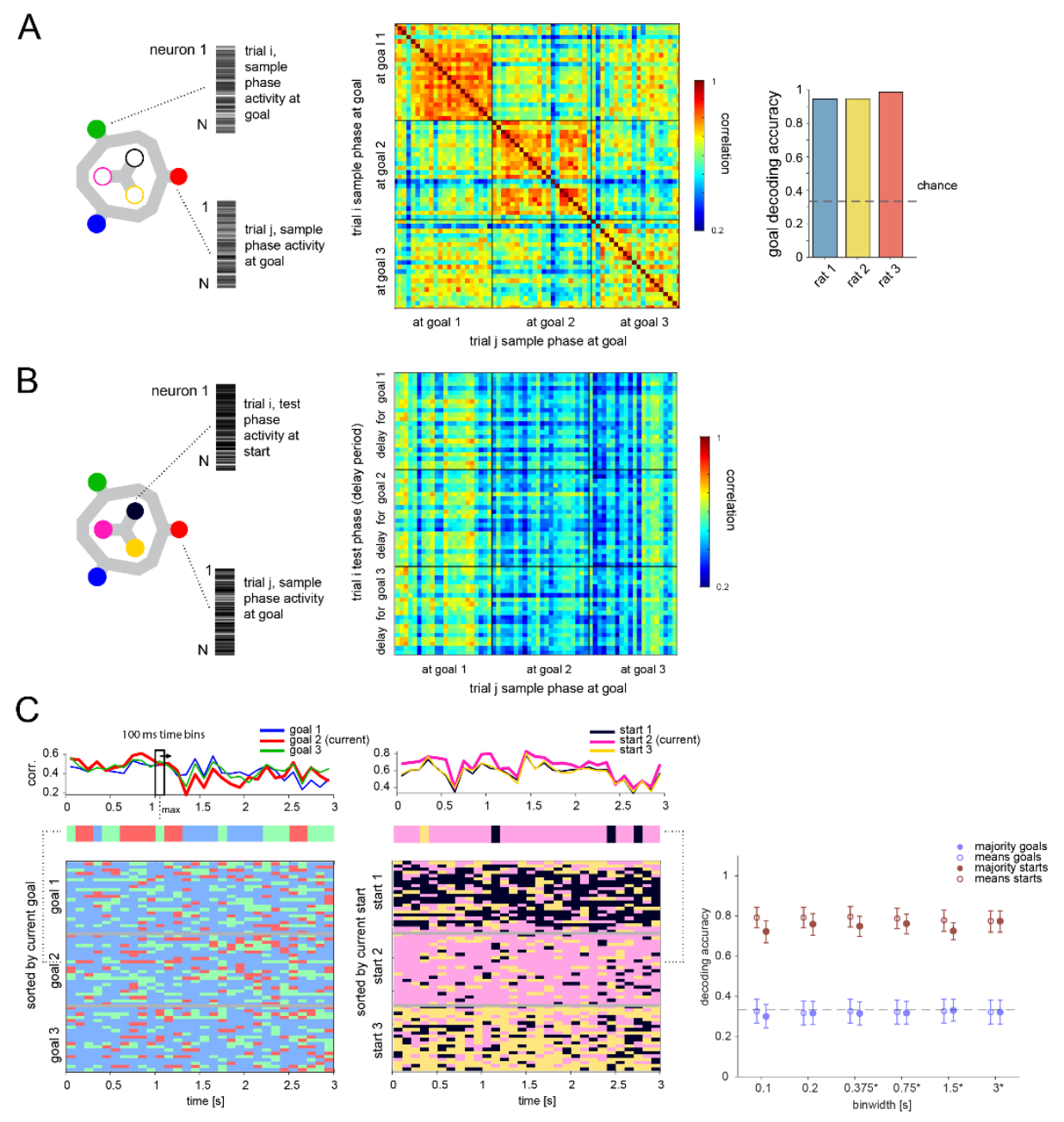

Figure 2. Memory is not maintained by goal-location-specific activity in the delay period.

(A) Population vectors (PVs) of activity while animal at each goal during sample phase (left) are distinct and stable (middle, correlation matrix of single trials in one animal; right, decoding accuracy, logistic regression classifier, mean over all trials and animals: $94.48 \%, 95 \%$ bootstrap CI: [90.87, 96.80]). (B) Correlation of PV while at goal during sample phase and PV during delay period while animal must maintain that goal-specific information. (C) Left, top: Example singletrial PV over time during delay period correlated with each goal PV. Left, middle: Goal with maximum correlation at each time bin above. Left, bottom: Same for all correct trials in this animal, sorted by current goal. Middle: Analogous to left but correlated with each start location PV (excluding contribution from current trial). Right: Correlation-based classification for range of binwidths (mean and 95\% CI). Class per trial determined by highest mean correlation over entire delay (unfilled) or majority vote of class with highest correlation at each time point (filled). 
bioRxiv preprint doi: https://doi.org/10.1101/2020.09.11.292888; this version posted September 11, 2020. The copyright holder for this preprint (which was not certified by peer review) is the author/funder, who has granted bioRxiv a license to display the preprint in perpetuity. It is made available under aCC-BY-NC-ND 4.0 International license.

*binwidths were 0.4, 0.8, 1.6 and $3.2 \mathrm{~s}$ for one animal that had 3.2 (versus 3 ) s delay (also for Figures $3 \mathrm{C}$ and $5 \mathrm{~A})$. 


\section{Figure 3}
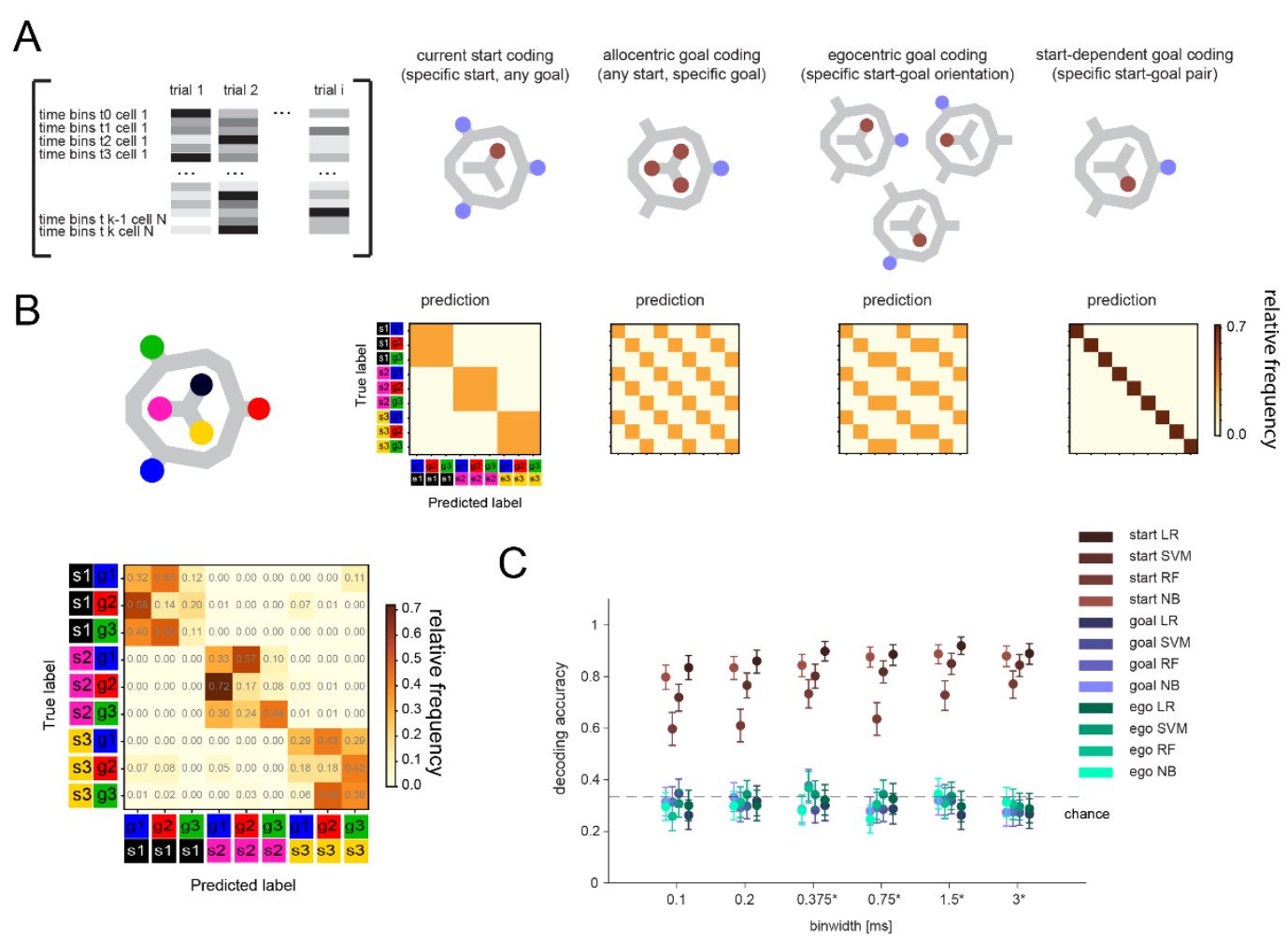

Figure 3. Lack of differential activity patterns corresponding to the current, remembered goal in the delay period.

(A) Leftmost: Differential patterns considered corresponded to activity across cells and time bins during delay period. Potential encoding schemes (left to right): start location represented independently of current, remembered goal; current goal represented independently of current (start) location; current goal represented in egocentric coordinates, i.e. direction to current goal with respect to current (start) location; current goal represented distinctly in different start locations. (B) Population activity analysis of potential encoding schemes during delay period using supervised classification. Top: Confusion matrices expected for each scheme. Bottom, left: Confusion matrix using support vector machine (SVM) classification ( $0.75 \mathrm{~s}$ bins) for one animal. (C) 3-class delay period activity classification using logistic regression (LR), SVM, random forest $(\mathrm{RF})$, or Naïve Bayes after feature selection (NB) over range of time resolutions (mean and 95\% CI). 


\section{Figure 4}
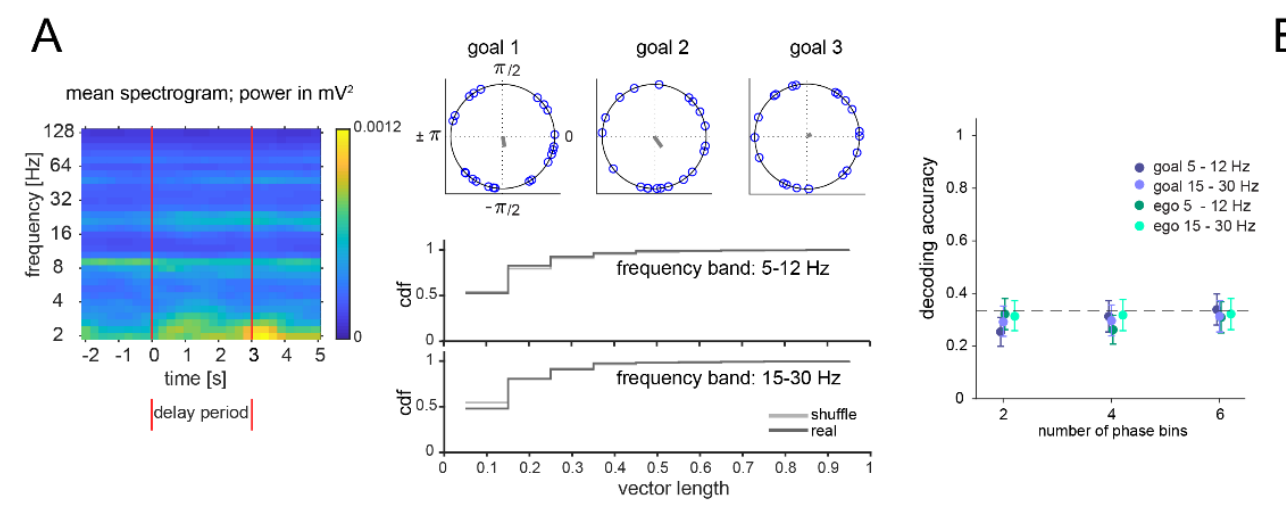

B

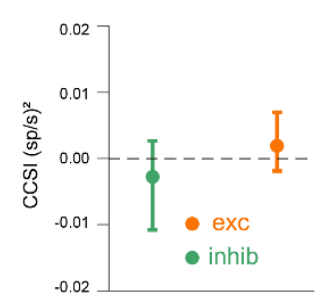

Figure 4. Lack of differential phase or covariance of firing corresponding to the current, remembered goal in the delay period.

(A) Phase analysis. Left: Spectrogram of delay period LFP. Middle: Example cell phase preference of delay period spikes and resultant vector length (r, gray) across all trials for each current goal (top). Cumulative distribution of $\mathrm{r}$ for all cells from one animal compared to shuffle of trials for two frequency bands (bottom). Right: Decoding accuracy (mean and 95\% CI) using spike counts at specific phases. Phases for each frequency band were divided into 2, 4 or 6 phase bins. (B) crosscorrelation selectivity index for the delay period (CCSI, after Barbosa et al., 2020 is a measure of the difference in covariance between trials where the current goal is the one where a given pair of neurons preferentially fires at during the sample period and trials where the current goal is either of the other two goals) for cell pairs determined to have excitatory or inhibitory interactions (mean and $95 \% \mathrm{CI}$, see methods). 


\section{Figure 5}

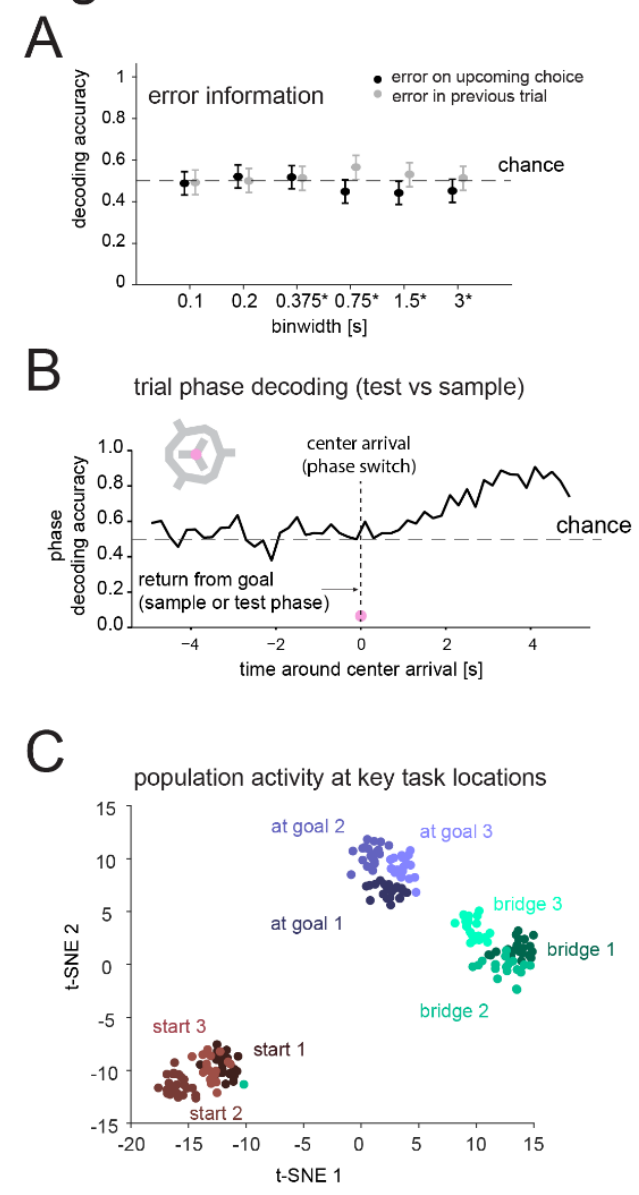

Figure 5. Prefrontal cortex encodes task-relevant information and forms groups of behavioral equivalence.

(A) Decodability of whether goal error occurred in upcoming or previous test phase based on population activity during delay period (mean and 95\% CI). (B) Decodability of task phase from population activity (200 ms bins) while animal is moving inbound from goal to center in sample or test phase (pre- $0 \mathrm{~s}$ ) and after it arrives at center, for one animal. Similar results in another animal (not shown). (C) t-SNE of population vectors of activity while animal is at key task locations: individual starts, goals, and bridges/routes. 


\title{
Methods
}

\author{
$\underline{\text { Experimental Procedures }}$
}

Surgery:

All procedures were conducted in accordance with the Janelia Research Campus Institutional Animal Care and Use Committee. The chronic Neuropixels implant surgery followed previously described methods (Jun et al., 2017). Briefly, animals were anesthetized with isoflurane and mounted in a stereotaxic frame (Kopf Instruments). After thorough cleaning of the skull, a ground screw was placed through the skull above the cerebellum. A small craniotomy (diameter $\sim 1 \mathrm{~mm}$ ) was made above the target area (AP: $3.24 \mathrm{~mm}$, ML: $0.6 \mathrm{~mm}$ ) in the right hemisphere. A single-shank Neuropixels " 1.0 " probe was lowered over the course of about an hour to a depth of 6.0-6.3 mm. The craniotomy was covered with artificial dura (Dow Corning Silicone gel 3-4680) and any parts of the probe outside of the brain were covered with sterile Vaseline. The probe was permanently fixed to the skull with dental acrylic and a protective cone made of copper mesh and dental acrylic or light cured cement was built around the probe. Recordings started after animals had fully recovered and were accustomed to the recording cable when attached to the implant, $\sim 2-$ 3 weeks after implant surgery.

Behavioral procedures:

Rats were housed in a reverse light cycle room (12 h:12 h day:night) and training and experiments were conducted mostly in the dark phase.

Rats learned the "multi-start/multi-goal/multi-route" ("MSMGMR") task over the course of several months in successive learning steps with generally one learning session per day. Animals were food restricted (with their weight maintained at $\geq 85 \%$ of their initial weight) to increase motivation to collect reward in our task.

Reward was given in the form of a sweet and nutritious liquid reward (Ensure Plus). The reward was dispensed from custom-made Teensy-operated reward pods which, along with the custom-made nose ports and bridges (that provide available "open" routes when up and are 
unavailable "blocked" routes when down in this elevated maze environment), were controlled by custom-written finite state machine software written in Matlab.

Data from three male Long-Evans rats were included in this study. (Two other animals were trained to high levels of performance for this study, but the recordings were lost before training was complete, in one case due to probe failure. In both of these cases, the probe was implanted before training began and the losses occurred after $\sim 4$ months of training. To limit such outcomes, the probe was implanted after training was complete in the subsequent animals (two of the three included ones).) Animals were $\sim 12$ weeks of age when training began for two animals and $\sim 6$ months for one animal.

To accustom animals to the elevated maze layout and the type of reward they would receive, animals were placed on the maze for $\sim 30$ min per session to explore and collect reward from any of the reward pods, which were located at the end of each goal arm and one in the center of the maze. All routes were available at this point in training and animals could freely move around to collect reward from any of these four reward sites on the maze (Figure 1A). Animals started exploring the maze immediately and learned to navigate between the reward sites within a few sessions.

Next, the sample phase of the task was introduced. Here, the animal learned the meaning of the visual cues (blue LEDs on the goal reward pod(s) blinking) and to find the current goal location by repeatedly being visually guided to the same goal and returning to the center after each run, which allowed it to understand the basic structure of the task (i.e. run out, run back to center, run out, and so on). This sample phase was implemented in two different configurations throughout the training and recording sessions for the three rats. For one rat, the correct goal location was indicated by a blinking light at the correct reward pod. For the other two rats, we used a reversed configuration where the correct goal was the only goal reward pod not blinking. This configuration was introduced to reduce the potential for the animals to remember the location of a simple visual cue instead of remembering the spatial location of the goal. However, both versions were readily learned by the animals and did not lead to any obvious changes in behavior in the test phase.

After the animal had learned to follow the guided cues, the test phase was introduced. Here the animal was cued 3-4 times to sample the same goal, followed by one run in which the animal was not cued and had to navigate to the same (correct) reward pod. If the animal went to an incorrect reward pod in the test phase first, they received a diminished or no reward if they then 
went to the correct one afterwards. The number of repeated 'sample phases' to the same goal was successively lowered until sample and test phases were interleaved.

Next, the use of a particular route was enforced during the sample phase. After animals returned from the test phase to the center reward pod, two of three bridges were lowered, i.e. the routes were blocked, forcing the animal to use the remaining one in the sample phase. The route available in each sample phase was chosen pseudorandomly.

Next, to introduce the nose pokes, all routes were blocked upon arrival of the rat at the center after the sample phase. The animal could choose any nose port; correct poking was indicated with a $4 \mathrm{kHz}$ tone upon brief poking $(50 \mathrm{~ms})$. After holding for the specified duration, all routes would become available (i.e. all bridges were raised). At this stage, the duration of the required poke was successively increased from the initial $50 \mathrm{~ms}$ to approximately 1 second. Once the animal developed a habit of choosing the same nose port to make the routes available, only one pseudorandomly chosen nose port would elicit the tone and raise the bridges. The nosepoke duration was then successively increased until the animal became proficient at holding it for 3 seconds (for two animals) and 3.2 seconds (for one animal). In two animals (the ones in which the goal was indicated as the pod that was not blinking), any incorrect nose poke was indicated by a constantly on light at that port after it had been poked at least once.

As a final learning step, only one route would become available after the correct nose port poke (i.e. for the test phase).

The final version of the task used the following pseudorandom method for determining the goal, available sample phase route, correct nose poke, and available test phase route for each trial. The pseudorandom sequence of trials was determined anew for each session. There are 27 distinct combinations of start position, goal location and test phase routes. To keep these combinations in balance overall and locally and to discourage formation of preferences for a particular goal, the order of these 27 combinations was randomly permuted with the constraints that (i) each of the three subblocks of nine trials was also balanced to equally contain each start location, goal location and test phase bridge, and (ii) the same goal was not repeated more than two consecutive trials. The sample route only had the constraint that in a subblock of nine trials, for each goal location each of the routes was presented once in the sample phase. Identical blocks of 27 trials were repeated in a given session. Note that there was no indication given to the animal of the 27-trial block or 9-trial subblock structure, so the entire session appeared as a single long sequence of 
trials. In this final version, the animal was not allowed to correct an error in the test phase and had to go to the center to initiate a new trial. In addition to the given pseudorandomly determined sequence of trials, if the animal made an error in the test phase of a trial, that same trial could be repeated one time (but with a potentially different sample route). In terms of discouraging preferences for a given goal, repeating a trial due to an error would necessarily mean the animal was not repeating a trip to the same goal in the test phase.

After the animal was able to perform the full task, training was continued until the animals reached $\sim 70 \%$ accuracy (total training time from naïve animal to this point was $\sim 3.5-6$ months). Then (for two animals), a Neuropixels probe was implanted in the medial prefrontal cortex (while for one animal the Neuropixels probe had been implanted before training). Recording began after the animal recovered and was acclimated to the recording cable.

Between training sessions, the maze was wiped with $70 \%$ ethanol to reduce any odor cues animals might use to navigate to the correct goal. Furthermore, the maze was mounted on a turnable-like frame and rotated to one of three orientations in between sessions, to further lower the probability that animals used local cues to remember goal locations. The maze was set up in a room with multiple cues outside of the maze, such as other lab equipment. Care was taken to ensure that the reward pods at the goals all delivered the same amount of reward and appeared visually identical. These precautions were taken to ensure that animals learned to use distal, non-local cues for navigation and to encode the currently rewarded goal location.

After conclusion of the experiments, animals were deeply anesthetized and underwent transcardial perfusion with saline followed by 4\% PFA for fixation. Brains were removed and sliced for histological verification of the recording site.

\section{Electrophysiology}

Neural data from Neuropixels "1.0" probes (https://www.neuropixels.org) was recorded with SpikeGLX software (http://billkarsh.github.io/SpikeGLX/). 384 channels were recorded simultaneously across subareas of the medial prefrontal cortex in two separate frequency bands (spike, $300 \mathrm{~Hz}$ to $10 \mathrm{kHz}$ sampled at $30 \mathrm{kHz}$, and LFP, $0.5 \mathrm{~Hz}$ to $300 \mathrm{~Hz}$ sampled at $2.5 \mathrm{kHz}$ ). The recording system and a laptop capturing the digitized data from the probe were mounted on a manually controlled, motorized rotating platform mounted at the ceiling to avoid the cable from 
becoming too twisted from the animals' turning. This apparatus was used for two of three animals. For one animal, the experiment was briefly interrupted to 'untwist' the cable by rotating the animal when it became necessary.

\section{$\underline{\text { Data Analysis }}$}

All data analysis was done using custom-written programs in Matlab or Python, and for some machine learning procedures the scikit-learn library was used (Pedregosa et al., 2011).

\section{Preprocessing:}

Multiple sessions were recorded from each animal but only one was included in the analysis per animal as the probes were not moveable and the population of cells could not be assumed to be independent across different recording sessions. JRCLUST (Jun et al., 2017) (version 08/2019, Vidrio) was used to automatically presort the spike data then manually curate it afterwards. To allow the animal to settle into the behavioral task and to remove global drifts leading to changes in firing rate across a significant number of cells (which were observed to occur at the beginning of each session, presumably due to the handling of the rat necessary to attach the probe to the cable), 10-20 trials were removed from the beginning of each session. Because we were searching for a working memory code that was stable and robust throughout the session, and to reduce the possibility that non-stationarities would reduce the generalization performance of the decoders, we selected for analysis the subset of cells that satisfied the following 3 stability/robustness criteria applied to each cell separately (but we also performed the main analyses including all cells without such selection, Figure S3-2). First, the overall firing rate had to be stable across the session: specifically, a linear regression on the standardized firing rate in 10 $\mathrm{s}$ bins over the session was performed and the absolute difference between the first bin and the last bin could not exceed 1 (i.e. the slope of any change in firing rate needed to be within \pm 1 s.d./n, where $\mathrm{n}=$ the total number of $10 \mathrm{~s}$ bins). Second, the firing rate in delay periods had to be stable across the session: specifically, the absolute difference of a linear regression on the summed spike count for all delay periods ( 3 or $3.2 \mathrm{~s}$ each) between the first and the last delay period could not exceed 1.4. Third, the cell needed to be active in a minimum number of delay periods: specifically, 
the cell had to fire at least one spike in at least one-sixth of all delay periods (set to potentially allow for a cell that was active in half of the delay periods for a particular goal and silent otherwise). The numbers of clusters isolated during spike sorting were 182, 131, and 98 for the three animals and, after applying the criteria above, 103, 86, and 72 cells remained. Putative fastspiking GABAergic interneurons were excluded from analysis based on having a combination of faster waveform (shorter peak to trough interval) and higher firing rate across the whole recording duration, resulting in 97, 84, and 68 active, stable, putative principal cells for the three animals.

Unless otherwise noted, only correct test phase trials were used for analysis. After removing the trials in the beginning as described above the numbers of total trials (number of correct trials) per animal were 79 (62), 81 (64) and 145 (110).

Correlation analysis:

For the correlation matrices in Figure 2A and B, a "goal arrival" population vector for each trial was calculated from the three second period after the animal had arrived at the goal in the sample phase (where time point 0 was assigned to be the time that the infrared beam on the reward pod was broken, which triggers delivery of the reward). Similarly, for all test phases a delay period population vector was calculated. The matrices containing the raw firing rates were concatenated and the Pearson's correlation coefficient was calculated for all combinations of population vectors. In Figure $2 \mathrm{~A}$ only the correlations among goal population vectors are shown and in Figure $2 \mathrm{~B}$ the correlation between delay period population vectors and goal population vectors are shown.

To test whether the remembered goal is preferentially represented in the delay period over time (Figure 2C), we calculated the average population vector from all sample phases when the animal was at one specific goal and correlated the resulting 3 population vectors with each time bin in all delay periods. For each delay period the 'winning' goal was either the one with the highest mean correlation with that goal across all bins or the one that had the highest number of time bins in which the correlation was highest with that goal (majority vote). A corresponding approach was taken to classify each delay period with regards to the start (current location), except the current delay period was excluded from the average of the start population vector. Here and elsewhere, a bootstrap analysis was used to calculate the $95 \%$ confidence interval of the decoding 
accuracy: for each binwidth, 10000 samples were drawn randomly from all trials from all animals with replacement and the 2.5 and 97.5 percentile values of the means were taken as the interval.

H-score analysis:

To assess what information is encoded in the delay period at the single-cell level (Figure S3-1), the spikes in each delay period were binned using a variety of binwidths and each cell $\times$ bin was considered one sample of a class. Several types of classes were considered separately: the current start (current location), the remembered goal, the goal in egocentric coordinates (i.e. behind the start, to the left, or to the right), or the combination of the start and the remembered goal $(3 \times 3$ classes). The distributions of spike counts of samples belonging to different classes (e.g. the different starts) were compared using the Kruskall-Wallis test. To correct for multiple comparisons, false discovery rate (FDR) correction was applied to each binwidth tested.

Supervised machine learning classification methods:

Generally, for each classification method, a range of hyperparameters were tested and a set of parameters that reached the highest cross-validation accuracy for start (i.e. current location) decoding was chosen for each method and kept constant. A leave-one-out cross-validation scheme was used for all classification methods. The numbers of samples per class were balanced throughout by randomly subsampling from the class(es) with the higher number of samples in the training set. Decoding accuracy was reported as the mean of the cross-validated accuracy over all classes. For population analyses where the delay period was binned in time (Figures 3B,C, S2-1B, S3-2 and 5A,B), all bins of a given delay period were concatenated into one vector and each cell $\times$ bin was treated as a separate feature, i.e. the activity patterns considered were fixed with respect to the starts of the delay periods (and analogously for the phase bins in Figure 4A). The matrix size used for classification was thus \# of time bins times * \# of cells by \# of trials. Each feature was standardized over all trials by subtracting the mean and dividing by the standard deviation, unless stated otherwise. Logistic regression (Figure 2A, right, 3C, 5A) was used with L2 regularization. The support vector machine classifier (Figure S2-1B, 3C, S3-2B and 5B) was used with a Gaussian kernel to allow for nonlinear decision boundaries. The kernel coefficient was set 
to 0.001 and L2 regularization was used. In Figure 3B, left bottom, the data was divided into 9 classes, corresponding to the 9 possible combinations of remembered goals and current start locations. For Figure 3C, three classes corresponding to either the three possible start locations, three allocentric goals, or three egocentrically defined goals were used. Correspondingly, for the analysis of task phase and spatial selectivity at the goal (Figure S2-1B; note the time period analyzed was the $3 \mathrm{~s}$ after the animal had arrived at the goal as in Figure 2), the data were divided into 6 classes corresponding to the 6 possible combinations of goal location and task phase. For the summary plot containing all animals in Figure S2-1B, middle, only the classification accuracy of either phase or goal was considered. For Figure S2-1B, right, two classifiers were trained, one with all data from the sample phase and one with all data from test phase. The remaining data were each used to predict the goal it encoded, i.e. data from the test phase were fed into the classifier built from sample phase data and the other way around. Decoding accuracy was given as the mean over all three classes. In Figure 5B for trial phase decoding at the center we only compared trials in which the animal took the same bridge back to center so that direction of arrival at the center was comparable.

For the Random Forest classification in Figure 3C and Figure S3-2, the data was prepared and balanced as described above, and the forest contained 1000 trees for each classifier. The maximum number of features considered for finding the best split was chosen to be $\sqrt{n}$, with $n$ being the number of features considered, i.e. for smaller time bins where the number of features is higher as described above, more features would be considered for each split.

For the Naïve Bayes classification (Figure 3C and S3-2), Gaussian distribution of the features was assumed and for each classifier only the $10 \%$ of features with the highest H-scores (from Kruskal-Wallis test) were used.

LFP-phase analysis (Figure 4A and S4-1):

LFP channels that corresponded to references or were noisy (standard deviation, std, either lower than 1/4 of the mean std or 4 times higher than the mean std) were removed. The LFP trace considered for a given cluster was the average of 10 LFP channels that were at least 8 sites away in both directions from the center of the cluster (i.e. the site with maximum amplitude) whose phase was analyzed, generally consisting of five sites above and below the center of the site (but 
if the center was too close to the edge of a block of recorded channels, the 10 channels used to average could be split unequally, e.g. 8 sites above and 2 sites below for a cluster near the bottom of a block). The LFP from $3 \mathrm{~s}$ before to $3 \mathrm{~s}$ after the delay period was filtered in the 5 to 12 , or 15 to $30 \mathrm{~Hz}$ (FIR filter), but only the delay period itself was considered for phase analysis. The phase of the oscillation of a frequency band was determined by calculating the angle of the Hilbert transform. Periods in which the resulting phase was not monotonically increasing between peaks were rejected (mean time rejected per delay period, across animals and both frequency bands: 206 ms, maximum time rejected: $1.322 \mathrm{~s}$, out of 3-3.2 s total) and spike times were mapped onto phases. For each cell the spike phases from all delay periods were divided into three classes, corresponding to the currently remembered goal. The length of the mean phase (resultant vector length) was computed as a measure of preferred firing phase for each cell and class. To test whether firing phase contained any information about the remembered goal location, the labels (remembered goal 1, remembered goal 2 or remembered goal 3) of delay periods were shuffled and the distribution of resultant vector lengths were compared to the one from the actual labels (Figures 4A, middle and S4-1). In a separate approach (Figure 4A, right), the phases of all spikes were binned into 2, 6 or 12 bins, corresponding to the number of spikes that were elicited in a particular phase bin (e.g. one of the bins for the 2-bin case included phases from -90 to $90 \mathrm{deg}$ ). All phase bins for each cell were concatenated and used as features for a logistic regression classifier trained on all but one test trial and tested on that trial (i.e. leave-one-out). To account for differences in total valid duration of each delay period (which could be less than the full duration due to the existence of periods with non-monotonically increasing phase), the counts in each bin in each delay period were divided by the total valid duration of that delay period. To account for differences in overall spike rate, these adjusted counts were normalized by subtracting the mean and dividing by the standard deviation over all delay periods for a given feature.

Covariance analysis (Figure 4B and S4-2):

Covariance was analyzed using the method described in Barbosa et al. (Barbosa et al., 2020) and the associated code at https://github.com/comptelab/interplayPFC. The following adaptations were made to fit our experimental data. Only cells that were significantly modulated at the sample goal location (i.e. different for different goals) were included in the analysis. A cell's 
'preferred goal location' was the one where it had the highest firing rate. Only pairs of neurons that shared the same preferred goal location were considered for analysis.

As in Barbosa et al. (Barbosa et al., 2020), spikes for all trials were binned in $10 \mathrm{~ms}$ bins and shuffled in steps of $50 \mathrm{~ms}$. The cross-covariance was calculated for each shuffle (1000) and the mean subtracted from each trial to remove any dynamics faster than $50 \mathrm{~ms}$. The resulting (jittercorrected) cross-covariance was taken to be the mean of the 3 bins around the 0 -lag bin. In the case of the single time point analysis the full delay period was considered ( 3 or $3.2 \mathrm{~s}$ ). For the time resolved version in Figure S4-2 bottom, time windows of $1 \mathrm{~s}$ were used and cross-covariance was repeatedly calculated in steps of $50 \mathrm{~ms}$. An 'excitatory pair' of neurons was considered as such if the sign of the mean jitter-corrected covariance was positive both for the preferred and nonpreferred trials and conversely considered an 'inhibitory pair' if the sign was negative for both. Pairs with inconsistent signs were discarded. The sign was calculated separately for the delay period only and the extended delay period in Figure S4-2 for the time resolved version ( $-2 \mathrm{~s}$ to 5 $\mathrm{s}$, with 0 being the beginning of the delay period).

This procedure resulted in the following numbers of total pairs/excitatory/inhibitory for the full delay period: rat $1: 288 / 67 / 67$, rat $2: 339 / 88 / 82$, rat $3: 232 / 61 / 59$.

For the time resolved version these numbers were: rat 1:288/72/59, rat 2:339/72/84, rat 3 : $232 / 62 / 66$.

The cross-correlation selectivity index (CCSI) (Barbosa et al., 2020) for the excitatory pairs was the mean difference of the cross-covariance in preferred and non-preferred trials, and similarly for the inhibitory pairs. The numbers of preferred and non-preferred trials were matched (by randomly subsampling the non-preferred trials).

t-distributed stochastic neighbor embedding (t-SNE) - analysis (Figure 5C and S5):

For t-SNE embeddings, firing rate population vectors from all delay periods (correct trials), the period 0-3 sec after goal arrival in the sample phase, and activity during crossing of the bridge after the delay period (crossing time: $0.28 \mathrm{~s}$ on average) in the test phase were embedded in 2dimensional space according to Maaten and Hinton (Maaten and Hinton, 2008); perplexity was set to 35 and the learning rate to 100 . Ten embeddings were calculated for each data set and the embedding with the lowest Kullback-Leibler divergence between data and embedding is shown. 
bioRxiv preprint doi: https://doi.org/10.1101/2020.09.11.292888; this version posted September 11, 2020. The copyright holder for this preprint (which was not certified by peer review) is the author/funder, who has granted bioRxiv a license to display the preprint in perpetuity. It is made available under aCC-BY-NC-ND 4.0 International license.

The overall structure of embeddings was stable over multiple runs and a range of perplexities and learning rates. Hyperparameters were kept constant when the embeddings were calculated separately for subareas. 


\section{Supplemental Figures}
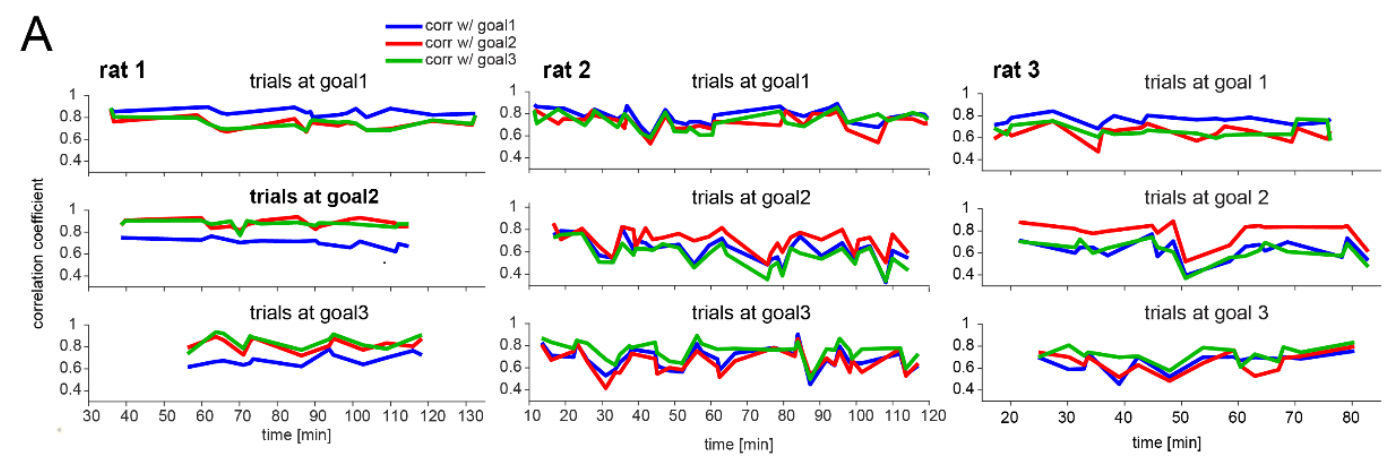

B
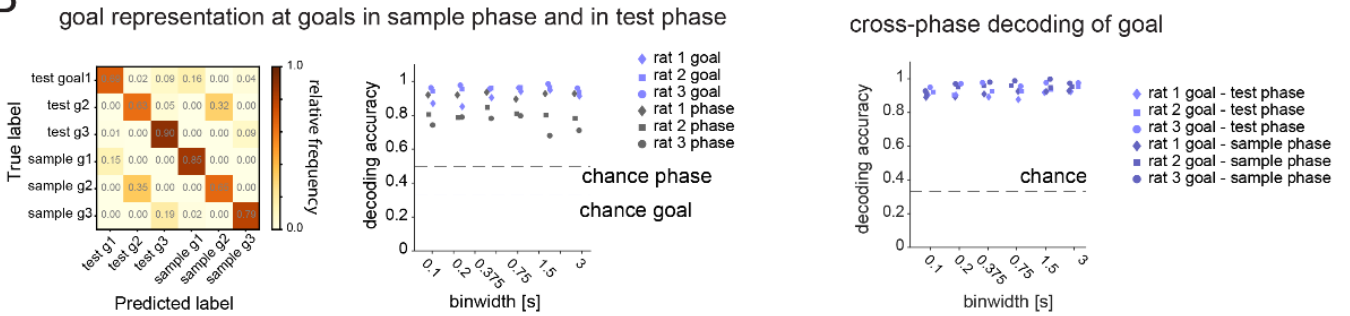

Figure S2-1. Representations are distinct and stable at goals.

(A) The average firing rate population vector at each goal (first $3 \mathrm{~s}$ after arrival) over the first 5 visits in the sample phase was calculated and each was correlated with the single visit population vectors of all following trials during the sample phase. The correlation coefficient was generally higher and at a similar level over the duration of recording sessions (90 - $135 \mathrm{~min})$ for the current goal location. (B) Sample and test goal decoding at goal. Left: SVM decoding confusion matrix for one animal (binwidth: $750 \mathrm{~ms}$ ); Middle: decoding performance for goal when at goal (regardless of phase, light blue) and trial phase (regardless of which goal, gray) for all animals at a range of binwidths. Note that both the identity of the goal as well as the trial phase in which it is visited can be decoded. Reward amount is smaller in sample than in test phase, which may contribute to the difference between test and sample phase activity at the goal. However, activity exhibits similarities at each goal across sample and test phases; Right: Decoding of goal identity as in Middle, but here the ability to predict the goal the animal is at is based on a classifier trained on data from the respective other task phase, thus assessing the overlap of goal representation during sample and test phases. Decoding accuracy was just as high as in the task phase-mixed classifier (Middle), suggesting no interference between task phase representation and spatial location code. 
cell counts (after selection for stability of firing rate throughout task performance):

infralimbic

rat 1: 27

rat 2: 21

rat 3: 18

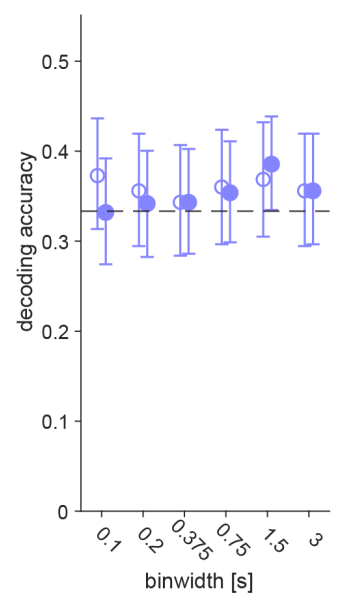

prelimbic

rat 1: 41

rat 2: 51

rat 3: 37

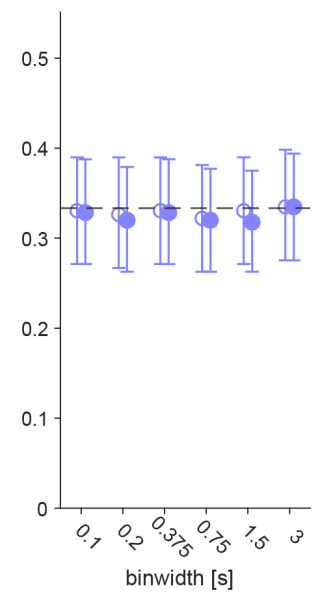

ACC

rat 1: 7

rat 2: 8

rat 3: 13

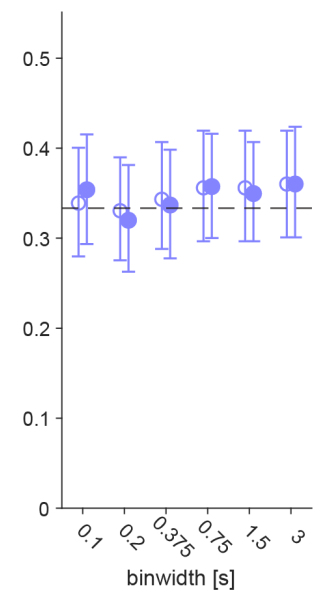

DP

rat 1: 22

rat 2: 4

rat 3: -

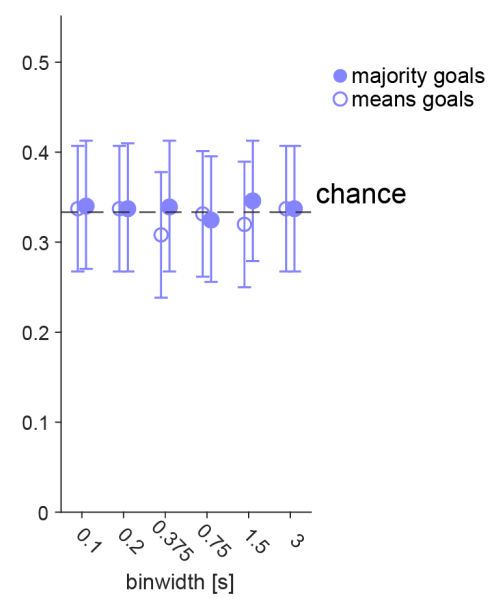

Figure S2-2. Goal-location-specific representations are not maintained in the delay period for any subarea.

Same analysis as in Figure 2C, right, but for subareas of prefrontal cortex separately: correlation scores for remembered goal location at different temporal binwidths (mean and 95\% CI). Remembered goal location could not be decoded in any subarea. 

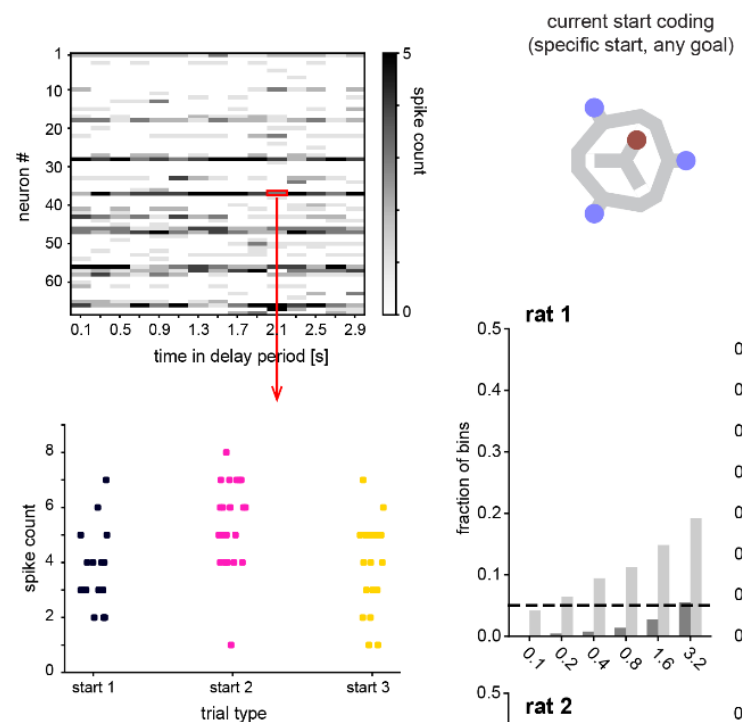

allocentric goal coding
(any start, specific goal)

egocentric goal coding (specific start-goal orientation)

start-dependent goal coding
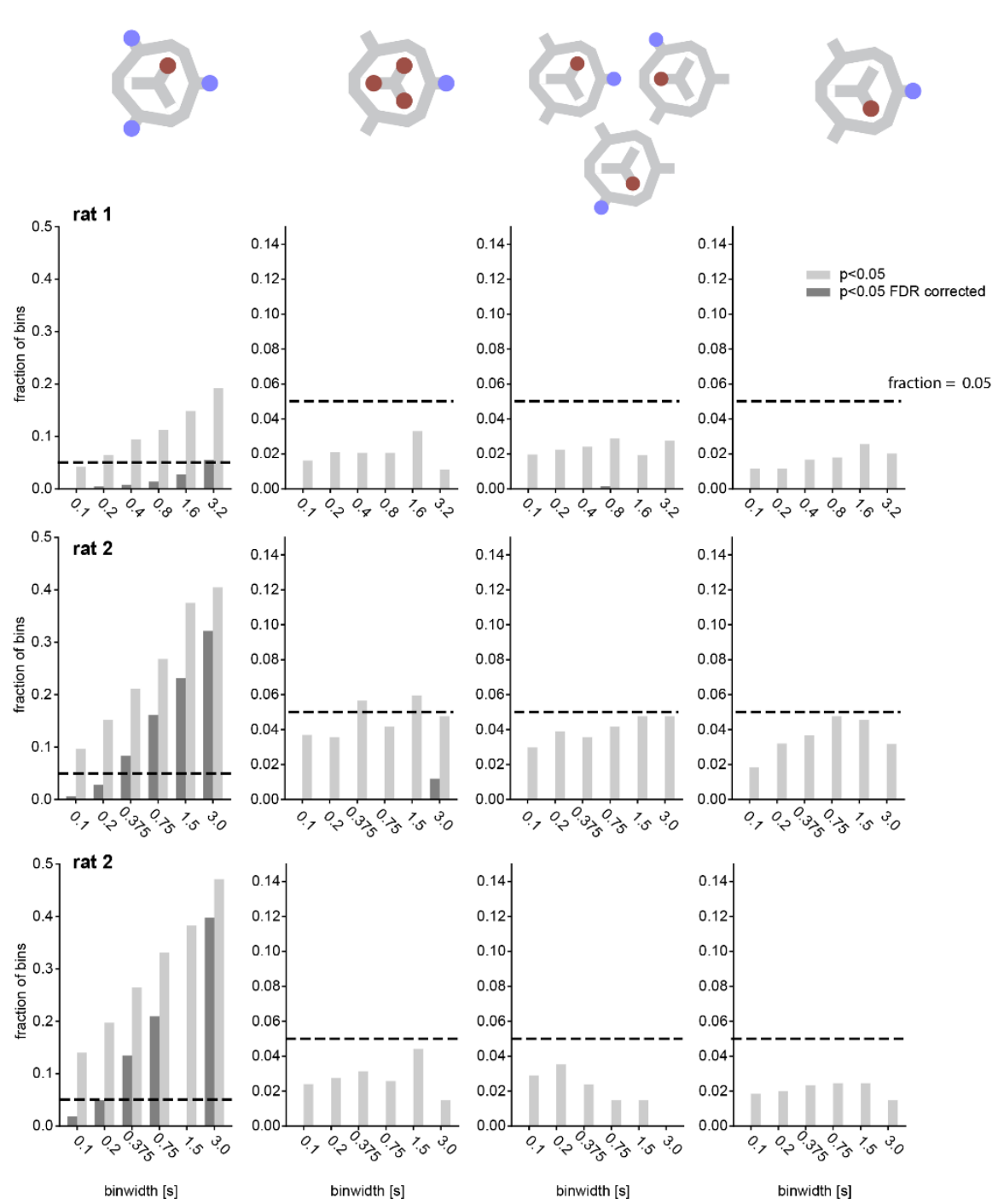

Figure S3-1. Single-cell firing rate analysis for individual animals.

Left Top: Example 200 ms-binned activity and test of significant encoding by single cell $\times$ bin (bottom). Middle Top: List of potential encoding schemes (analogous to Figure 3). Bottom: Corresponding fraction of cell $\times$ bins with Kruskal-Wallis p-value $<0.05$ (light gray, dark gray: FDR corrected). Note that for all animals a fraction of cell $\times$ bins encoded the current start position but not the maintained goal location. 


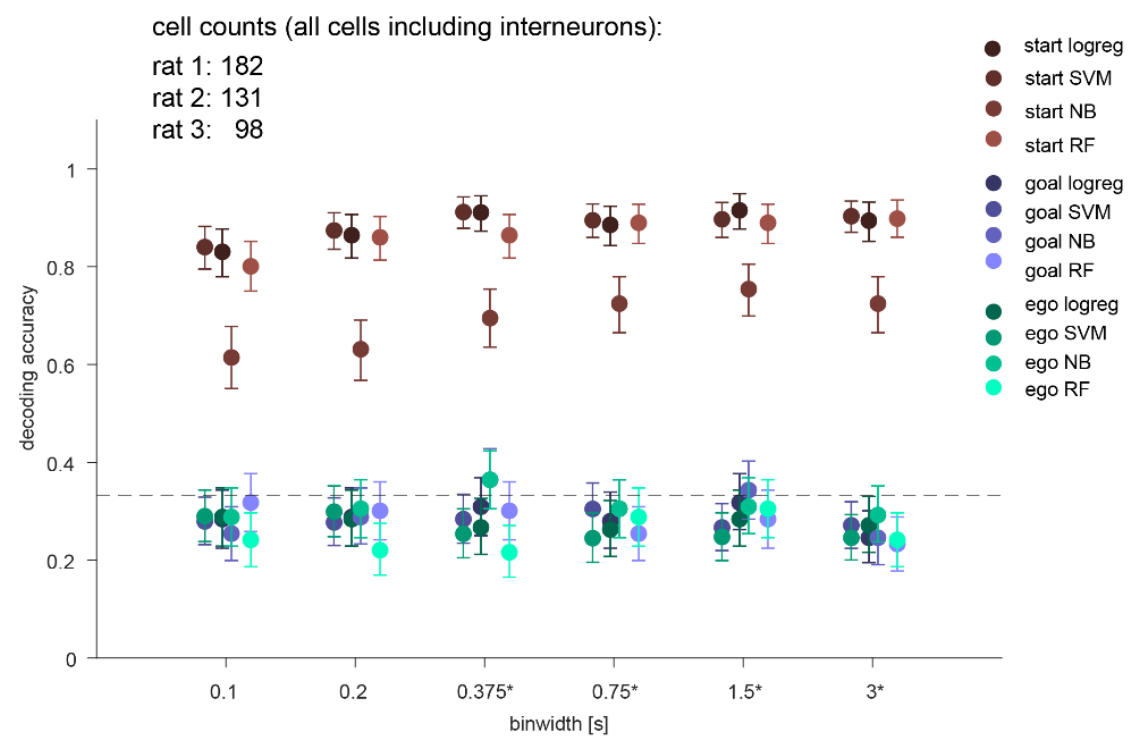

Figure S3-2. Population decoding for all cells independent of selection criteria.

Summary of 3-class classification of delay period activity using different methods using all cells recorded (same as Figure 3C but without stability selection criteria (see methods) and including interneurons; logistic regression (LR), SVM, random forest (RF), Naïve Bayes after feature selection (NB)). Means across all animals and 95\% CIs are shown. 
bioRxiv preprint doi: https://doi.org/10.1101/2020.09.11.292888; this version posted September 11, 2020. The copyright holder for this preprint (which was not certified by peer review) is the author/funder, who has granted bioRxiv a license to display the preprint in perpetuity. It is made available under aCC-BY-NC-ND 4.0 International license.
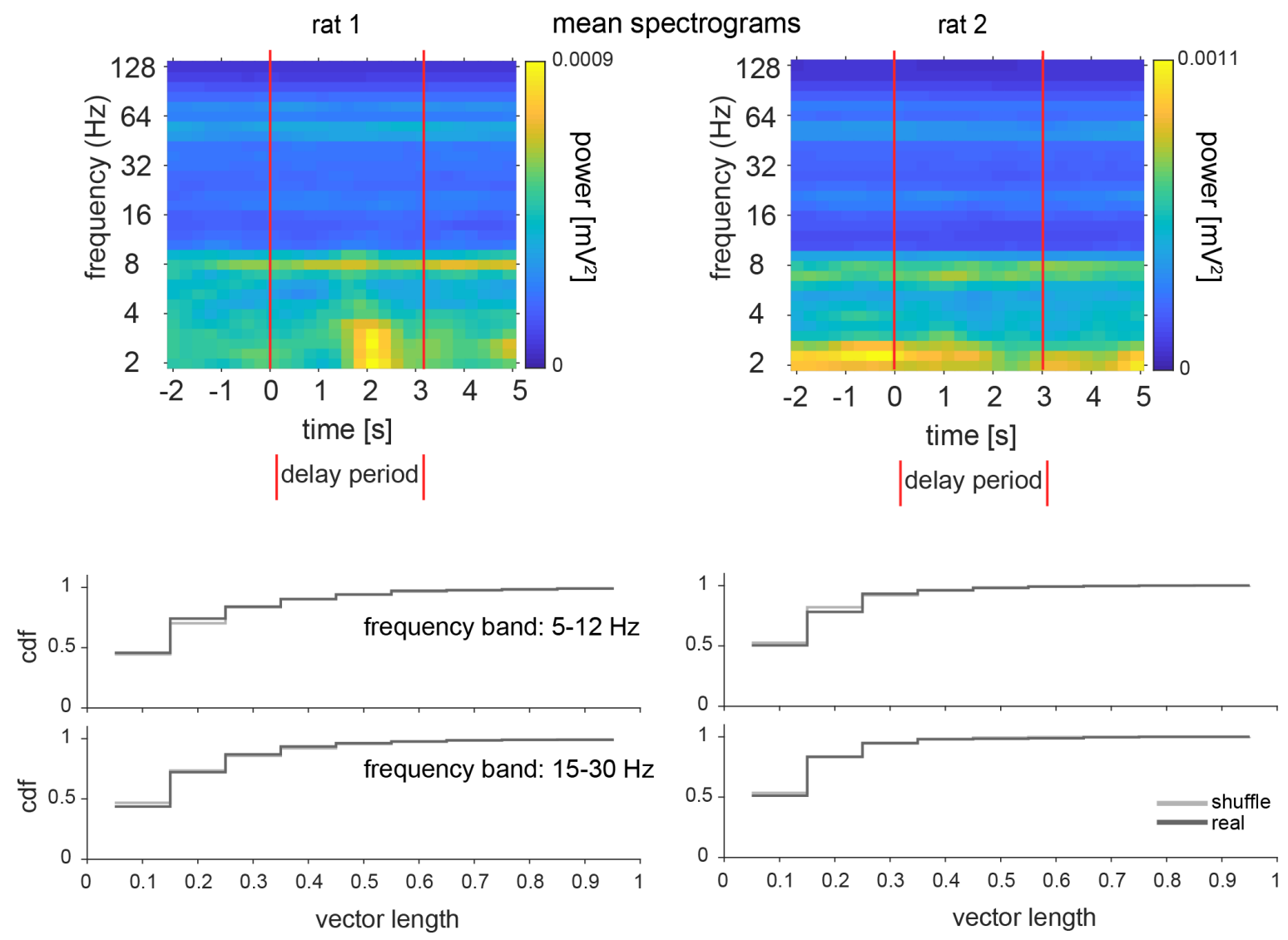

Figure S4-1. Phase analysis for individual animals.

Same as Figure 4A left and middle but for rat 1 and rat 2. 

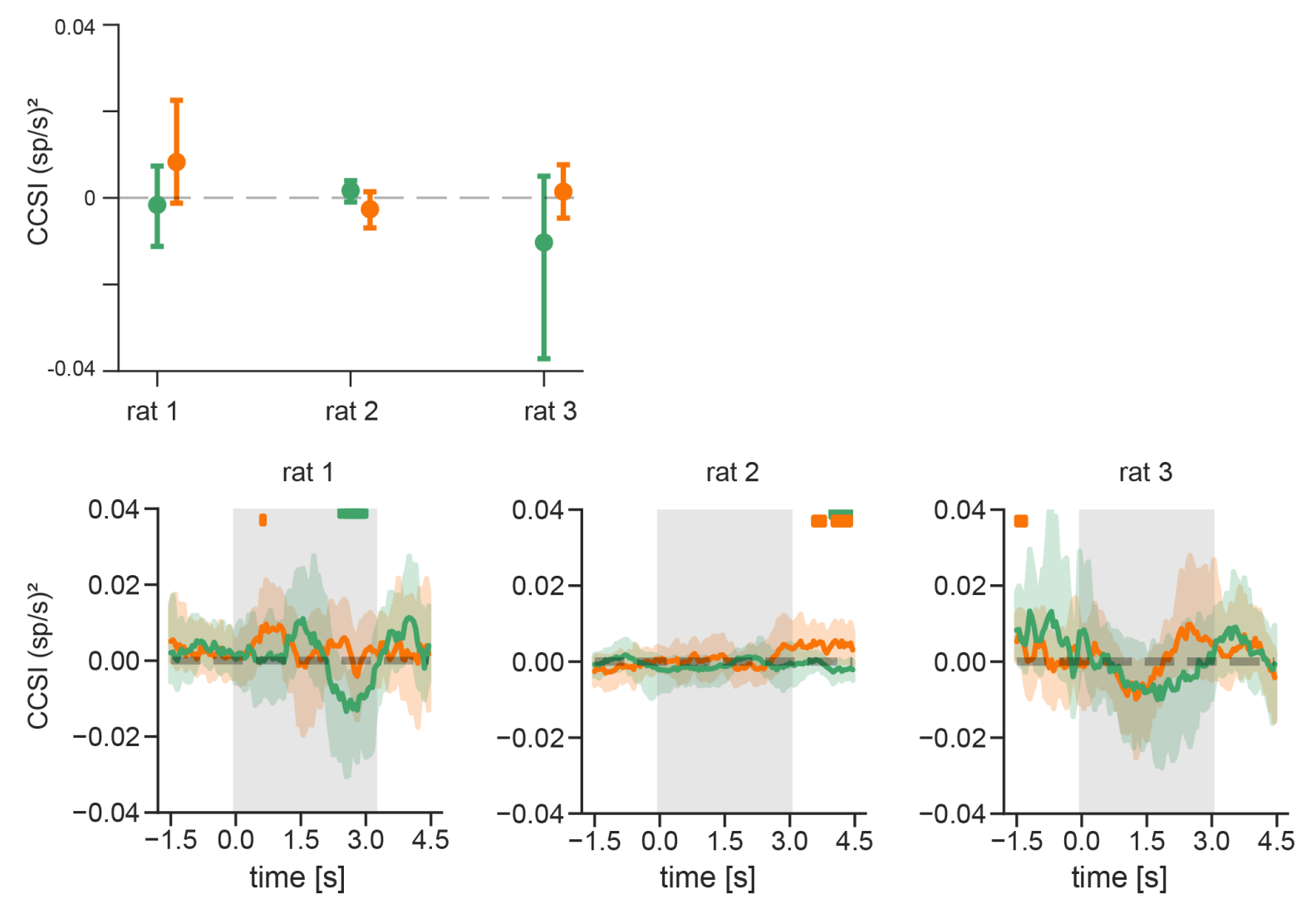

Figure S4-2. Covariance analysis for individual animals.

Top: CCSI for the full delay period for individual animals (mean and 95\% CI). Bottom. Time course of CCSI (in sliding $1 \mathrm{~s}$ windows) for individual animals. Gray shaded area depicts the delay period. Green: inhibitory pairs. Orange: excitatory pairs. Plotted are means and 95\% CIs. Green and orange horizontal segments represent centers of individual windows where the mean covariance differed for preferred and non-preferred trials; however, the CCSIs for the full delay period above (and pooled across animals in Figure 4B) show that there is no overall significant relationship between excitatory or inhibitory neuron pair covariances and the current goal in the delay period. 
all stable cells

ACC
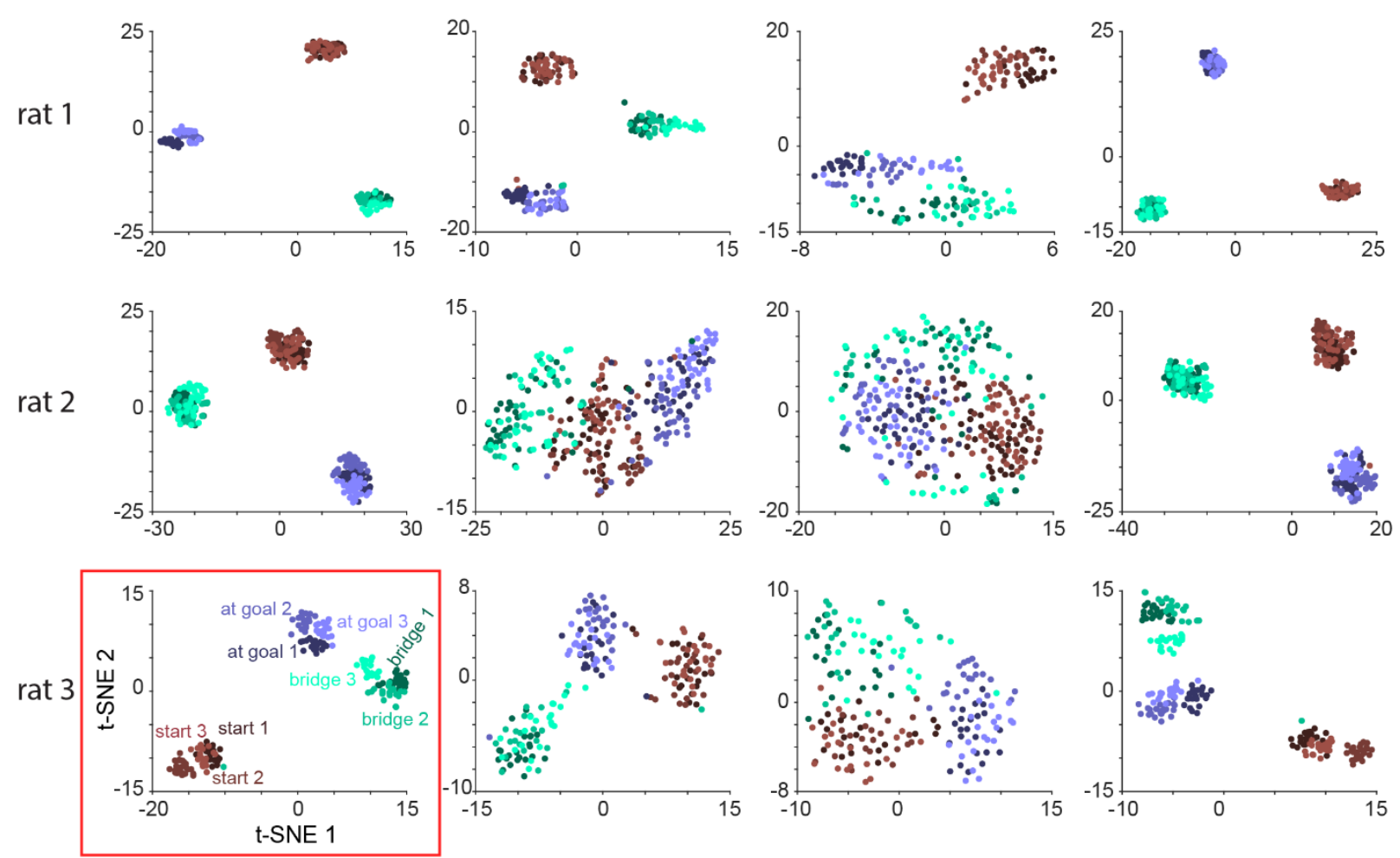

Figure S5. t-SNE analysis for each animal and subarea.

Same as Figure 5C but for cells belonging to ACC, prelimbic cortex, and infralimbic cortex separately. Only cells with stable firing rates were considered (see methods). Numbers of cells for each subarea as in Figure S2-2. Red rectangle: same as shown in Figure 5C.

\section{Movie S1.}

Video showing three consecutive trials of animal performing the task. 